\title{
The Nobel Prize in Physiology or Medicine 2019 How Cells Sense and Adapt to Fluctuating Oxygen Levels
}

\author{
Agnishwar Girigoswami* \\ Kelambakkam, Chennai, Tamilnadu, INDIA \\ Corresponding author - Dr. Agnishwar Girigoswami (agnishwarg@gmail.com) \\ Chettinad Health City Medical Journal 2019; 8(4): 136 - 137 \\ DOI: https://doi.org/10.36503/chcmj 8(4)-07
}

*Professor, Medical Bionanotechnology, Faculty of Allied Health Sciences, Chettinad Academy of Research and Education (CARE),

Since the onset of modern biology, the fundamental importance of oxygen for the conversion of useful energy from food by animal cells has been well understood. The unknown part was the mechanism on how the animal cells adapt when the level of oxygen changes. William G. Kaelin Jr., Sir Peter J. Ratcliffe and Gregg L. Semenza have introduced the concept and the molecular machinery that regulates the gene expression in response to oxygen level variations. For this important contribution to the science, Prof. Thomas Perlmann, secretary of Nobel Committee for Physiology or Medicine announced on 7th of October, 2019 that the Nobel Assembly at the Karolinska Institute has decided to award the Nobel Prize in Physiology or Medicine 2019 to William G. Kaelin Jr., Sir Peter J. Ratcliffe and Gregg L. Semenza jointly. ${ }^{1}$

Mitochondria which are present in the animal cells use atmospheric oxygen for the enzymatic production of useful energy from food. This concept of enzymatic process was introduced by Professor Otto Warburg, the recipient of Nobel Prize in Physiology or medicine in 1931. Therefore, the mechanism of adequate supply of oxygen to the tissues and cells are important. The carotid body holds specialized cells that sense the oxygen level in blood. The 1938 Nobel Laureate, Professor Corneille Heymans discovered the mechanism by which carotid body controls our respiratory rate, sensing the blood oxygen level. Also, there are other physiological adaptations involved in addition to the rapid adaptation controlled by the carotid body to hypoxia. These key physiological responses in hypoxia include increased levels of erythropoietin hormone (EPO) that induces erythropoiesis for the increased production of red blood cells. The hormone-controlled erythropoiesis was known, but the mechanism by which oxygen level controls the process was unknown.

Dr. Semenza and Dr. Ratcliffe were studying separately the EPO gene and its regulations by changing the level of oxygen using gene-modified mice in early 1990s. Specific DNA segments located next to the EPO gene were shown to mediate the response in hypoxic conditions. ${ }^{2}$ Sir Peter Ratcliffe was also concentrating on oxygen dependent regulation of the EPO gene at the same time and both the research groups found that the oxygen sensing mechanism was present in all tissues including kidney cells where EPO is normally produced. Further, Dr. Semenza wanted to identify the cellular components involved in this response process. He discovered a protein complex, the hypoxia-inducible factor (HIF) in cultured liver cells that binds to the identified DNA segment in an oxygen dependent manner. In 1995, Semenza identified the gene sequence which is responsible for the HIF encoding. ${ }^{3}$ The protein HIF is a dimeric protein complex that plays an integral role in hypoxia consisting of two different DNA binding proteins - HIF-1 $\alpha$ and ARNT (aryl hydrocarbon receptor nuclear translocator). At lower oxygen level, the amount of HIF-1 $\alpha$ increases and regulates the expression of EPO gene after binding to the DNA segments. Researchers have observed that the incidence of normal degradation of HIF-1 $\alpha$ ceases in hypoxia. HIF-1 $\alpha$ gets degraded by a protein complex called proteasomes in normoxia (Figure 1).

When Dr. Semenza and Dr. Ratcliffe were exploring the regulation of EPO gene at the same time Dr. Kaelin was working on von Hippel-Lindau's (VHL) disease, an inherited syndrome that lead to increased risk of certain cancers in families with inherited VHL mutations. Dr. Kaelin observed that the $\mathrm{VHL}$ gene encodes a protein that prevents cancer. He also observed that the VHL was involved in controlling responses to hypoxia. ${ }^{4}$ Finally Ratcliffe demonstrated successfully that VHL can physically interact with HIF-1 $\alpha$ which is important for the degradation at normal oxygen condition. Then the question was how oxygen level regulates the interaction between $\mathrm{VHL}$ and $\mathrm{HIF-1} \alpha$. At this point, they started to concentrate on the specific portion where

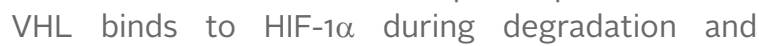
suspected that the key oxygen sensor is residing somewhere in the protein domain. In 2001, Kaelin and Ratcliffe demonstrated that in normoxia, hydroxyl groups are added to two specific positions in HIF-1 . $^{5}$ This prolyl hydroxylation helps VHL to recognize HIF-1 $\alpha$ for binding and rapid degradation of HIF-1 $\alpha$ at normal oxygen level. In this process, oxygen sensitive enzymes, prolyl hydroxylases are involved. Later, Ratcliffe also identified the role of 


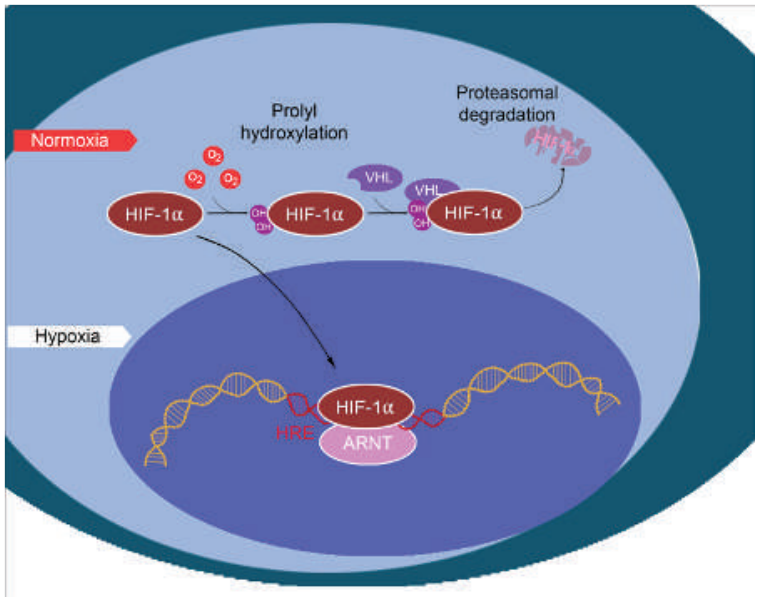

Figure 1: At normal oxygen level, oxygen regulates the degradation of HIF-1a by the addition of hydroxyl groups to HIF-1a in the presence of VHL, whereas in hypoxia, HIF-1a is protected from degradation. It accumulates in the nucleolus to associate with ARNT and binds to the specific DNA segment (HRE) in hypoxia regulated gene.

prolyl hydroxylases in oxygen sensing mechanism. ${ }^{6}$ This is how Nobel Laureates have established oxygen sensing mechanism. Their discovery has shown the new path for the development of new strategy to fight anemia, cancer and many other related diseases.

William G. Kaelin Jr. is an American physician scientist. He was born in 1957 in New York, USA. He received his medical degree from Duke University and took special training in internal medicine and oncology from Johns Hopkins University and Dana-Farber Cancer Institute in Boston. He became a full professor at Harvard Medical School in 2002. The Kaelin Laboratory studies the functions of tumor suppressor proteins including the retinoblastoma protein (pRB), the von Hippel-Lindau protein ( $\mathrm{pVHL})$, and a homolog of the p53 tumor suppressor protein called p73.

Sir Peter J. Ratcliffe is a British physician scientist. He was born in 1954 in Lancashire, UK. He received his degree in medicine from Gonville and Caius College at Cambridge University before his specialization in nephrology at Oxford. He became full professor at the University of Oxford in 1996. He is the Director of Clinical Research at Francis Crick Institute, London. He is known for his research into the regulation of erythropoietin, a hormone that stimulates red blood cell production in response to low blood oxygen levels, and for his research into the mechanisms cells use to sense oxygen.
Gregg Leonard Semenza is an American professor on genetic medicine at the Johns Hopkins School of Medicine. He was born in 1956 in New York, USA. He completed his undergraduate in biology from Harvard University, Boston before receiving MD/Ph.D. degree from the University of Pennsylvania, School of Medicine, Philadelphia. He became a full professor at the Johns Hopkins University in 1999. His research includes protection of the heart against ischemia-reperfusion injury, Role of HIF-1 in cancer, Gene and stem cell therapy for ischemic cardiovascular disease, Molecular mechanisms of oxygen homeostasis and Genetic syndromes due to mutations in genes encoding transcription factors.

\section{References}

1. Organisation NP. Press release: The Nobel Prize in Physiology or Medicine 2019.

2. Semenza GL, Nejfelt MK, Chi SM, Antonarakis SE. Hypoxia-inducible nuclear factors bind to an enhancer element located 3'to the human erythropoietin gene. Proc Natl Acad Sci U S A. 1991;88(13):5680-4.

3. Wang GL, Jiang B-H, Rue EA, Semenza GL. Hypoxia-inducible factor 1 is a basic-helix-loophelix-PAS heterodimer regulated by cellular $\mathrm{O}_{2}$ tension. Proc Natl Acad Sci U S A. 1995;92(12): 5510-4.

4. Maxwell PH, Wiesener MS, Chang G-W, Clifford SC, Vaux EC, Cockman ME, et al. The tumour suppressor protein VHL targets hypoxia-inducible factors for oxygen-dependent proteolysis. Nature. 1999;399(6733):271-5.

5. Ivan M, Kondo K, Yang H, Kim W, Valiando J, Ohh $M$, et al. HIFa targeted for VHL-mediated destruction by proline hydroxylation: implications for O2 sensing. Science. 2001;292(5516):464-8.

6. Jaakkola P, Mole DR, Tian Y-M, Wilson MI, Gielbert J, Gaskell SJ, et al. Targeting of HIF-a to the von Hippel-Lindau ubiquitylation complex by O2-regulated prolyl hydroxylation. Science. 2001;292(5516):468-72. 